\title{
Evaluation of Planetary Boundary Layer Scheme Sensitivities for the Purpose of Parameter Estimation
}

\author{
JOHN W. NIELSEN-GAMMON \\ Department of Atmospheric Sciences, Texas A\&M University, College Station, Texas \\ XiaO-Ming Hu And Fuging Zhang \\ Department of Meteorology, The Pennsylvania State University, University Park, Pennsylvania \\ JonAthan E. Pleim \\ National Exposure Research Laboratory, U.S. Environmental Protection Agency, Research \\ Triangle Park, North Carolina
}

(Manuscript received 25 November 2009, in final form 14 March 2010)

\begin{abstract}
Meteorological model errors caused by imperfect parameterizations generally cannot be overcome simply by optimizing initial and boundary conditions. However, advanced data assimilation methods are capable of extracting significant information about parameterization behavior from the observations, and thus can be used to estimate model parameters while they adjust the model state. Such parameters should be identifiable, meaning that they must have a detectible impact on observable aspects of the model behavior, their individual impacts should be a monotonic function of the parameter values, and the various impacts should be clearly distinguishable from each other.

A sensitivity analysis is conducted for the parameters within the Asymmetrical Convective Model, version 2 (ACM2) planetary boundary layer (PBL) scheme in the Weather Research and Forecasting model in order to determine the parameters most suited for estimation. A total of 10 candidate parameters are selected from what is, in general, an infinite number of parameters, most being implicit or hidden. Multiple sets of model simulations are performed to test the sensitivity of the simulations to these 10 particular ACM2 parameters within their plausible physical bounds. The most identifiable parameters are found to govern the vertical profile of local mixing within the unstable PBL, the minimum allowable diffusivity, the definition of the height of the unstable PBL, and the Richardson number criterion used to determine the onset of turbulent mixing in stable stratification. Differences in observability imply that the specific choice of parameters to be estimated should depend upon the characteristics of the observations being assimilated.
\end{abstract}

\section{Introduction: Parameters and parameter estimation}

Appropriate treatment of vertical mixing is an essential component of meteorological and air quality models. Planetary boundary layer (PBL) schemes are used to parameterize the vertical turbulent fluxes of heat, momentum, and constituents such as moisture within the PBL as well as in the free atmosphere. The accuracy of

Corresponding author address: John W. Nielsen-Gammon, Dept. of Atmospheric Sciences, Texas A\&M University, 3150 TAMUS, College Station, TX 77843-3150.

E-mail: n-g@tamu.edu the PBL scheme is critical for forecasts of local thermally and mechanically driven flows and air quality, and it also affects forecasts of larger-scale meteorological phenomena (Hacker and Snyder 2005). Errors and uncertainties associated with PBL schemes remain one of the primary sources of inaccuracies in model simulations (Pleim 2007b; Hu et al. 2010a).

Parameter estimation offers a way to improve the accuracy of parameterizations such as PBL schemes. Parameter estimation is a technique for determining the best value of certain model parameters through data assimilation or similar techniques. When applied to parameterizations of meteorological processes, one hopes to identify optimal parameter values within a given parameterization, 
with "optimal" defined over some appropriate domain in space and time.

For the specific application of optimizing a PBL scheme, the parameters to be estimated are not necessarily limited to numerical constants that appear explicitly in the parameterization formulation. For example, one could create a superparameterization, in which vertical mixing is computed as a weighted average of the mixing produced by various PBL schemes, and the weighting values would be the targets of parameter estimation. Alternatively, one could expand the set of estimable parameters within a single parameterization to allow for structural changes to the parameterization itself.

The set of possible parameters to be estimated is infinite. Consider a simple parameterization at grid point $i$ of $y_{i}$ in terms of $x_{i}$ :

$$
y_{i}=A x_{i}
$$

Structurally, this is a linear approximation. But one may generalize it as a power series in which there are infinite parameters:

$$
y_{i}=\sum_{j=-\infty}^{\infty} A_{j} x_{i}^{j},
$$

or as a nonlocal approximation over $N$ grid points:

$$
y_{i}=\sum_{j=1}^{N} A_{i j} x_{j},
$$

or as a function of various model variables:

$$
y_{i}=A_{i x} x_{i}+A_{i v} v_{i}+A_{i u} u_{i}+A_{i T} T_{i} \ldots
$$

The assertion that (1) is an optimal parameterization is equivalent to the assertion that all but one of the $A$ s in (2)-(4) are optimally set equal to zero. In principle, all of the $A$ s in (2)-(4), and other parameters besides, are hidden or implicit parameters that are also candidates for parameter estimation.

The optimization problem for parameter estimation may be defined locally or globally. Global parameter estimation involves the search for a single parameter value that performs best in all situations. Local parameter estimation allows for optimal parameters to be functions of space and time, in keeping with the idea that optimal parameters are likely to be flow or situation dependent. For example, the exponent in the formulation of boundary layer scaling of vertical eddy diffusivity [used in the Yonsei University (YSU) and Asymmetrical Convective Model, version 2 (ACM2) PBL schemes] is dependent on stability (Troen and Mahrt 1986). Parameter estimation permits not just optimization of a parameterization, but optimal evolution of a parameterization.

Advanced data assimilation methods [e.g., variational approaches and versions of the ensemble Kalman filter (EnKF)] are capable of extracting from observations significant information about the model parameters in addition to the model state. They can be used to counter model errors due to incorrect parameters by calibrating those parameters simultaneously with the model state during the analysis process. Parameter estimation using data assimilation methods has been a common approach to deal with model error associated with incorrect parameters (Navon 1997; Aksoy et al. 2006a,b; Zupanski and Zupanski 2006; Tong and Xue 2008; Kondrashov et al. 2008). In atmospheric sciences, variational data assimilation methods are traditionally used for parameter estimation. Only recently have ensemble-based schemes emerged as a promising method for parameter estimation (for a review, see Aksoy et al. 2006a).

The inverse problem of parameter estimation is essentially a problem of mapping from the space of model outputs (which is measurable) to the space of parameters. The mapping in EnKF is realized through the covariance between parameters and model outputs calculated from the ensemble (i.e., EnKF adjusts parameters using observations based on the covariance between them). However such mapping may fail under some conditions: (i) the changes produced by parameter variations do not project sufficiently strongly onto observation space, thus measurement errors can lead to large changes in estimated parameter values; (ii) the model output does not vary smoothly with the parameter to be estimated, thus the optimal parameter value may never be found; or (iii) various parameters have indistinguishable effects on model output, thus the wrong parameters may be adjusted. Navon (1997) groups all three conditions under the general term of identifiability, while Zupanski and Zupanski (2006) refer to (i) as observability and reserve the term identifiability for (ii) and (iii). Here, we will refer to (i) as observability, (ii) as simplicity, and (iii) as distinguishability. Thus, successful parameter estimation requires that the set of parameters to be estimated produce sufficiently large, well-behaved, and unique sensitivities in model output.

The objective of our research program is to use EnKF to estimate the optimal values of some fundamental parameters in the ACM2 PBL scheme in the Weather Research and Forecasting (WRF) model and improve the simultaneous state estimation. As a necessary first step (Tong and Xue 2008) in this program, this paper reports on a detailed sensitivity analysis to identify the best parameters to be estimated in ACM2. Such a sensitivity analysis enables us to rank a subset of chosen parameters according to their chances to be correctly 
identified in parameter estimation and help us understand the EnKF results (estimation of both parameters and state). Such a comprehensive sensitivity analysis is also useful for understanding the characteristics and sources of systematic errors of the ACM2 scheme and other similar PBL schemes, and may facilitate future improvements in PBL schemes of a similar type. The overall approach is applicable to any complex parameterization scheme.

The paper is organized as follows. In section 2, the ACM2 PBL scheme is briefly described and potentially identifiable parameters in ACM2 are summarized. Section 3 describes the model setup and diagnostic approach. In section 4, model sensitivities to each parameter are examined and related to physical causes. Section 5 discusses the numerical results in the context of parameter identifiability, seeking to identify the best parameters for parameter estimation. The paper concludes with a brief summary.

\section{Description of the ACM2 scheme and its potentially identifiable parameters}

The ACM2 PBL scheme (Pleim 2007a,b) includes an eddy diffusion component in addition to the explicit nonlocal transport of the original ACM1 scheme (Pleim and Chang 1992). A weighting factor is used to govern the portion of mixing due to local diffusion and nonlocal transport. The inclusion of a local eddy diffusion component leads to a more realistic representation of the shape of the vertical profiles of model variables near the surface (Pleim 2007a). For stable or neutral conditions, the portion of mixing due to nonlocal transport is set to zero, thus the ACM2 scheme transits to use pure local eddy diffusion to handle vertical mixing. The potentially identifiable parameters in ACM2 as implemented in WRF version 3 are discussed in the following paragraphs. For a full description of the ACM2 scheme and definitions of all variables, see Pleim (2007a,b). We discuss here only those formulas and variables that are essential for understanding the nature of the potentially identifiable parameters or that are different in the WRF implementation of ACM2.

For the local vertical eddy diffusion, the maximum of two methods of eddy diffusivity $K_{z}$ calculation (i.e., a PBL scaling form of $K_{z}$ and a local formulation of $K_{z}$ ) is applied. The PBL scaling form of $K_{z}$ within the boundary layer may be written [after Pleim (2007a), his Eq. (12)] as

$$
K_{z}(z)=k \frac{u_{*}}{\phi} z(1-z / h)^{p},
$$

where $k$ is the von Kármán constant (well known to within about $10 \%$ and therefore not very adjustable), $\phi$ is the similarity profile function (with different symbols for heat $\phi_{h}$ and momentum $\phi_{m}$ ), $z$ is the height above ground level, and $h$ is the height above ground level of the top of the boundary layer (PBLH). The exponent $p$ is a hidden parameter; Eq. (12) of Pleim (2007a) uses the value " 2 " rather than the symbol $p$. The value of $p$ partly determines the magnitude of the diffusivity, with smaller values leading to stronger diffusivity, and partly determines the level at which the diffusivity is a maximum. When $p=1$, diffusivity peaks in the middle of the boundary layer; the diffusivity maximum moves progressively lower for larger values of $p$. Troen and Mahrt (1986) consider values ranging from 1 to 3 for this parameter.

In the ACM2 implementation in WRF, $\phi_{m}$ is used for computing the friction velocity $u_{*}$, but $\phi_{h}$ is used in (5) for computing the vertical mixing coefficient $K_{z}$ for momentum as well as for temperature and mixing ratios. In earlier tests, little difference was found in computing a separate $K_{z}$ for momentum.

The universal functions $\phi_{h}$ and $\phi_{m}$ have been the subject of considerable research, and a variety of formulations exist (Foken 2006). For unstable conditions, a fairly general representation of the relationship between the two universal functions is

$$
\phi_{h}=P \phi_{m}^{2}
$$

Here $P$ is a hidden parameter. The ACM2 scheme uses $P=1$ (Pleim 2007a), but other values are possible and affect the local value of the Prandtl number. According to Foken (2006), the physical range of $P$ is small, perhaps $0.95-1.35$. A suitable range for $P$ is $0.9-1.5$.

For stable conditions, the profile functions of $\phi_{h}$ and $\phi_{m}$ are given (Pleim 2007b) as

$$
\phi_{h}=\phi_{m}=1+r \frac{z}{L}
$$

while for very stable conditions $(z / L>1)$ they are given as

$$
\phi_{h}=\phi_{m}=r+\frac{z}{L}
$$

Pleim (2007b) uses 5 for the value of the hidden variable $r$. According to Foken (2006), the presently accepted value is $r=6$, so it would be reasonable to allow $r$ to range from 4.5 to 7 .

The local formulation of $K_{z}$ in the ACM2 scheme takes several forms depending on the value of the local Richardson number Ri:

$$
\mathrm{Ri}>\mathrm{Rc}: K_{z}=K_{z o},
$$




$$
\begin{gathered}
0<\mathrm{Ri}<\mathrm{Rc}: \quad K_{z}=K_{z o}+\left|\frac{\partial U}{\partial z}\right|\left(1-\frac{\mathrm{Ri}}{\mathrm{Rc}}\right)^{2} l_{s}^{2}, \\
\mathrm{Ri}<0: \quad K_{z}=K_{z o}+\left[\left(\frac{\partial U}{\partial z}\right)^{2}(1-j \mathrm{Ri})\right]^{1 / 2} l_{s}^{2},
\end{gathered}
$$

where

$$
\begin{aligned}
l_{s}^{2} & =\left(\frac{k_{z} \lambda}{k_{z}+\lambda}\right)^{2} \\
K_{z o} & =V K_{v} \Delta z+(1-V) K_{c} .
\end{aligned}
$$

Here we have corrected transcription errors in Pleim [2007b, his Eqs. (4) and (5)] and written a generalized form for (11) and (13). The ACM2 value of $j$ is 25 (not 0.25 as stated in Pleim 2007b), but this parameter, arising only in cases of absolute instability, is not expected to be observable. The local Richardson number Ri includes the effects of moisture and is compared to a critical Richardson number Rc for identification of the stability regime. The ACM2 value for $\mathrm{Rc}$ is 0.25 , with a plausible range of values from 0.2 to 1.0. The parameter $\lambda$ is the asymptotic value of the turbulent length scale. It is set to $80 \mathrm{~m}$ in the ACM2 scheme, but is not well constrained and may be taken to vary from 40 to $120 \mathrm{~m}$.

The current WRF (3.1) implementation of the ACM2 scheme has $K_{z o}=K_{v} \Delta z$, which, in the context of (13), means that hidden parameter $V=1$. In this implementation $K_{v}$ depends on vertical resolution. A previous implementation has $K_{z o}=K_{c}$, which corresponds to $V=0$. The formulation in (13) allows parameter estimation of $V$ to determine which of the two formulations is most appropriate. ACM2 has $K_{v}=0.001$. It is sufficiently poorly known that it is plausible to allow it to range over an order of magnitude or more. Parameter estimation of $K_{c}$ is probably not possible when $K_{v}$ and $V$ are being estimated because of distinguishability issues.

A weighting factor of $f_{\text {conv }}$ is used to control the portion of mixing due to the nonlocal transport (Pleim 2007a):

$$
f_{\text {conv }}=\left(1+\frac{1}{k 0.1 a} \frac{u_{*}}{w_{*}} \frac{\phi_{h}}{\phi_{m}^{2}}\right)^{-1} .
$$

Here $w_{*}$ is the conventional convective velocity scale. The adjustable constant is $0.1 a$, and observations of the vertical profile of temperature should directly affect the proper value of $0.1 a$. The full plausible range of $0.1 a$ is between 0 and infinity, with 0 corresponding to fully local mixing and infinity corresponding to fully nonlocal mixing. The latter situation reduces to the ACM1 scheme (Pleim and Chang 1992). In ACM2, 0.1 $a=0.72$. The fraction of similarity functions in (14) reduces to $P$, but in our tests we keep the value of this fraction at 1 in (14). Thus, all variations in the specified fraction of nonlocal mixing are subsumed into parameter $0.1 a$.

The ACM2 scheme is sensitive to the diagnosed height of the top of the boundary layer ( $h$, also known as PBLH). PBLH is involved in the calculation of both local and nonlocal mixing. The height of the PBL top $h$ is diagnosed as the level at which the bulk Richardson number, calculated from the ground up under stable conditions and from the top of the convectively unstable layer under unstable conditions, equals a critical Richardson number $\mathrm{Ri}_{\text {crit. }}$ The designation of stable versus unstable conditions depends upon $h$, the Monin-Obukhov length, and the lapse rate between the lowest two model levels. The top of the convectively unstable layer is identified where the potential temperature equals the potential temperature of a buoyant plume originating from the surface. In general, a larger $\mathrm{Ri}_{\text {crit }}$ corresponds to a larger $h$ and greater exchange between the free atmosphere and the PBL. In ACM2 the value of $\mathrm{Ri}_{\text {crit }}$ is set to 0.25 . The plausible range of values of $\mathrm{Ri}_{\text {crit }}$ is $0.2-1.2$, corresponding on the low end to an assumption of a finite amount of time for turbulence to develop in the face of instability and on the high end to turbulence producing a stable profile rather than a neutral one. Note that the parameter $\mathrm{Ri}_{\text {crit }}$ is a criterion for a bulk Richardson number and is used only in the definition of $h$, while Rc, appearing in (9)-(11), is a criterion for a local Richardson number and is used to determine the stability regime. Thus, it is not inconsistent to allow $\mathrm{Ri}_{\text {crit }}$ and $\mathrm{Rc}$ to vary independently.

The potential temperature of a buoyant plume (used in PBLH calculations above) is (Pleim 2007a)

$$
\theta_{s}=\theta_{v}\left(z_{1}\right)+b \frac{\left(\overline{w^{\prime} \theta^{\prime}}\right)_{0}}{\left(u_{*}^{3}+0.6 w_{*}^{3}\right)^{1 / 3}} .
$$

The first term on the right-hand side is the virtual potential temperature of the lowest model layer, and the numerator is the surface heat flux (Pleim 2007a). The excess virtual temperature is sensitive to the scaling factor $b$ for the heat flux, with larger values of $b$ corresponding to larger excess buoyancy. Holtslag and Boville (1993) use $b=8.5$, and this value is adopted in ACM2, but as the thickness of the lowest model layer decreases the magnitude of the excess buoyancy relative to the lowest model layer should also decrease. Thus, $b$ could potentially be much smaller than 8.5 , and a plausible range would be from 0 to 10 . As $b$ becomes small, so does the height of the top of the PBL, $h$. 
TABLE 1. Potentially identifiable ACM2 parameters

\begin{tabular}{llccl}
\hline \hline & Parameter name & ACM2 value & Plausible range & \multicolumn{1}{c}{ Role of parameter } \\
\hline$p$ & & 2 & $1-3$ & Structure of local mixing within PBL \\
$P$ & Prandtl No. & 1 & $0.9-1.5$ & Nominal ratio of momentum/heat diffusion \\
$0.1 a$ & & $0-72$ & $0.2-1.2$ & Controls proportion of nonlocal mixing \\
$\mathrm{Ri}_{\text {crit }}$ & Critical Richardson No. & 0.25 & $0-10$ & Affects calculation of height of PBL \\
$b$ & & 8.5 & $4.5-7$ & Controls excess buoyancy of surface plumes \\
$r$ & & 5 & $0.2-1.0$ & Affects stable mixing in dimensionless profile \\
$\mathrm{Rc}$ & Critical Richardson No. & 0.25 & $40-120 \mathrm{~m}$ & Governs flow dependence of stable turbulence \\
$\lambda$ & & $80 \mathrm{~m}$ & $0-1$ & Asymptic value of turbulent length scale \\
$V$ & & 0.001 & $0.0003-0.006$ & Pormulation for $K_{z o}$ \\
$K_{v}$ & & & Proportional to minimum $K_{z}$ as function of \\
& & & & layer thickness \\
\hline
\end{tabular}

Table 1 summarized the complete list of potentially identifiable parameters discussed above. Together, the set of parameters affects unstable and stable mixing and has the potential to significantly alter the performance of the ACM2 scheme. The next step is to run an ensemble of simulations with these variables chosen within their full plausible range and to determine experimentally the nature of the sensitivity of the WRF scheme to each of these parameters. Then, a final decision may be made on which parameters to estimate through data assimilation.

\section{Experimental design}

Three model domains are run with one-way nesting. Figure 1 shows the domain configuration. The grid spacings are 108, 36, and $12 \mathrm{~km}$, respectively. The coarse domain covers North and Central America, the second covers the contiguous United States and most of the Gulf of Mexico, and the inner covers Texas and adjacent areas. All model domains have 43 vertical layers, and the model top is set at $50 \mathrm{hPa}$. The lowest model eta levels are at $1.000,0.996,0.990,0.980,0.970,0.960,0.950,0.940,0.930$,

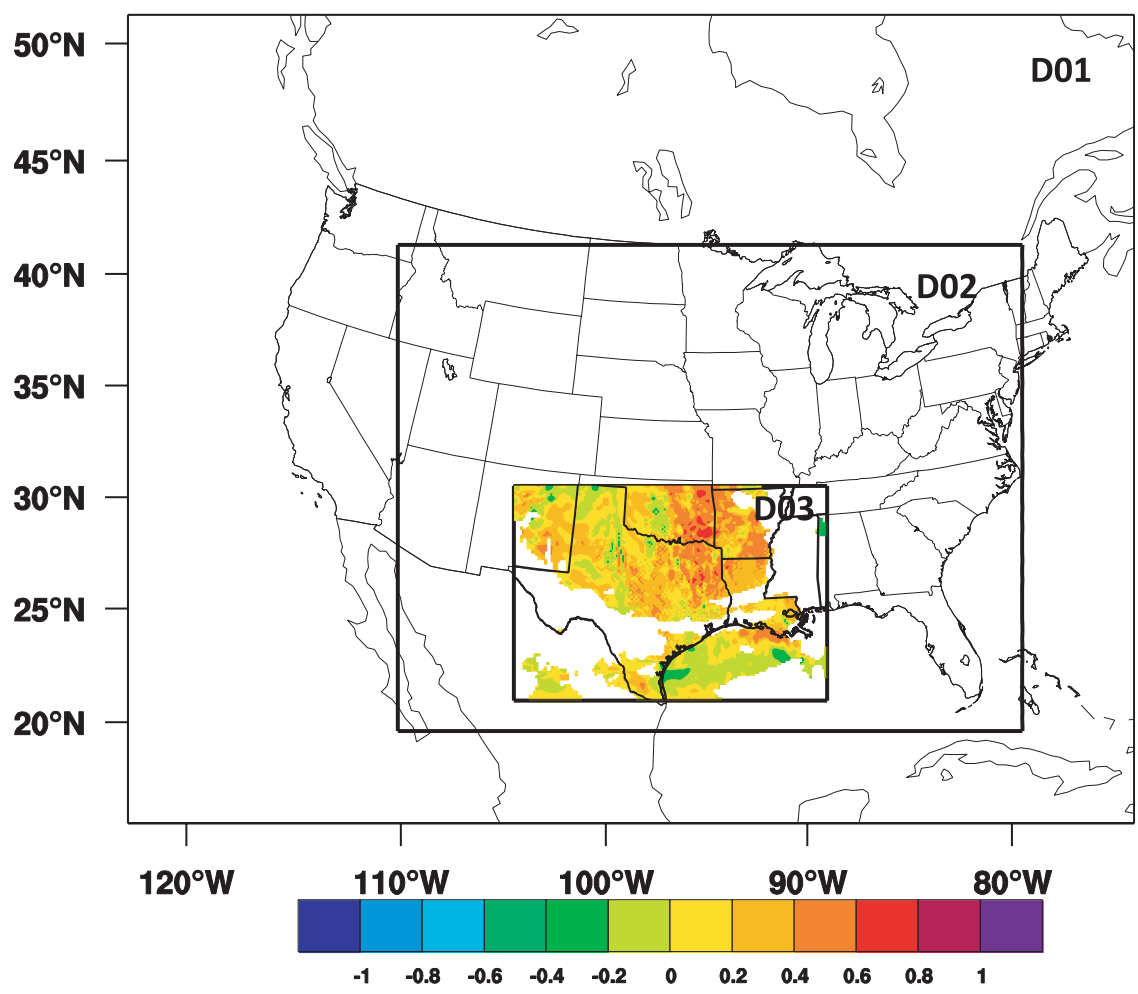

FIG. 1. Domain configuration and correlation between surface temperature and $K_{v}$ at $0000 \mathrm{CST}$ 31 Aug 2006 over the no-precipitation area in domain 3. 
0.920, 0.910, 0.895, 0.880, 0.865, 0.850, 0.825, and 0.800 . All model domains use Dudhia shortwave radiation (Dudhia 1989), Rapid Radiative Transfer Model (RRTM) longwave radiation (Mlawer et al. 1997), the WRF Single-Moment 6-Class Microphysics scheme (WSM6; Hong et al. 2004), the Noah land surface scheme (Chen and Dudhia 2001), the ACM2 PBL scheme, and the Monin-Obukhov surface layer scheme. The National Centers for Environmental Prediction (NCEP) Global Forecast System (GFS) operational analyses and forecasts are used for initial and boundary conditions.

The model start time is 0000 UTC 30 August 2006 (1800 CST 29 August) and the model run length is $48 \mathrm{~h}$. During this period, a ridge of high surface pressure extended southward into northeast Texas. Winds were generally northerly in eastern Texas and southerly in western Texas, with a weak sea-breeze circulation near the coast and a southerly Great Plains low-level jet over the Texas Panhandle during the second night. Skies were mostly clear, except for daytime boundary layer cumulus and clouds associated with some west Texas thunderstorms. The period falls within an air quality field program known as the Second Texas Air Quality Study (TexAQS II), and high concentrations of ozone were observed in eastern Texas on both days (Parrish et al. 2009).

Two sets of deterministic simulations are conducted to test the model sensitivities to 10 parameters in the ACM2 scheme listed in Table 1. In one set, all parameters are set to their default except for one parameter, which is assigned one of five values (evenly distributed within its specified range). A total of 50 WRF model runs are performed in this set, called the single-parameter set. In the other set, all potentially identifiable parameters are assigned random values within their range of variability. A total of 50 WRF model runs are performed in this set, called the multiparameter set.

The EnKF does not know about physical constraints on model parameters. In order that these parameter sensitivity simulations are as similar as possible to our future parameter estimation simulations, a technique is developed and implemented that constrains the model parameters to lie within the physically realistic ranges specified in Table 1. For each model parameter $x$, we create a normal parameter $y$. Each normal parameter $y$ is related to $x$ by

$$
\begin{aligned}
& y=\tan \left[\pi\left(\frac{x-A}{B-A}-\frac{1}{2}\right)\right], \\
& x=A+\left[0.5+\frac{\arctan (y)}{\pi}\right](B-A) .
\end{aligned}
$$

With this formulation, $y$ varies from $+/-$ infinity while $x$ varies within the range $[\mathrm{A}: \mathrm{B}]$. Parameter estimation

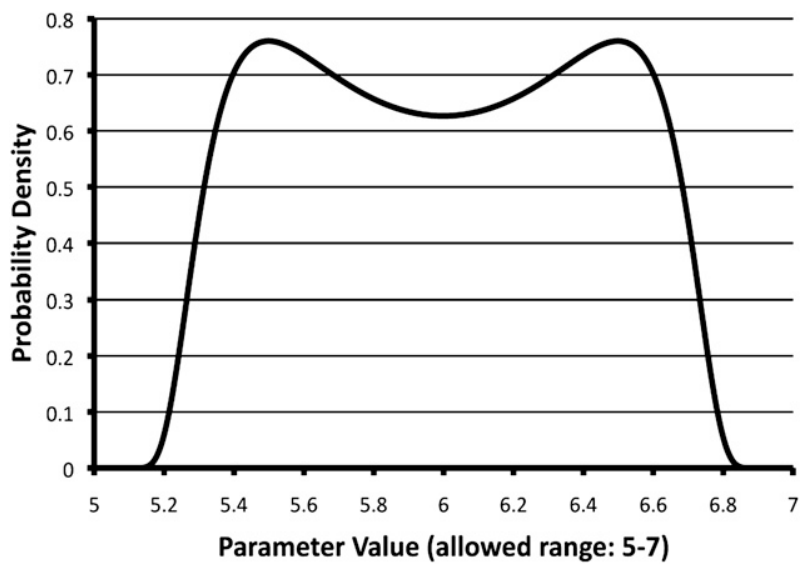

FIG. 2. Probability distribution of an arbitrary parameter allowed to vary from $A=5$ to $B=7$, when transformed from a standard normal distribution using (16).

will be performed on $y$, and $y$ will be transformed to $x$ prior to its use in ACM2. In the multiparameter simulations, 50 pseudorandom values drawn from a normal distribution with mean 0 and 1 standard deviation are generated for each normal parameter $y$. Those 50 pseudorandom values are then transformed to the specific range of each parameter using (17). The transformation has been designed such that these initial pseudorandom values, when transformed into model parameters, populate about $70 \%$ of the specified ranges of those parameters with a fairly flat distribution (Fig. 2).

Alterations to the PBL parameterization produce both direct impacts on the vertical structure of model variables and indirect impacts on the evolution of meteorological phenomena such as moist convection or sea breezes. Surface-based moist convection, for example, is sensitive to PBL parameterization schemes, and the consequences of PBL-scheme-induced differences in simulated convection can propagate upscale to affect larger phenomena (Jankow et al. 2005; Nielsen-Gammon et al. 2005). Such convection would in turn alter the boundary layer characteristics beyond what was produced directly by the PBL scheme. Likewise, the intensity, timing, and inland penetration of simulated sea breezes are sometimes, but not always, affected by the boundary layer structures generated by different PBL schemes (Miao et al. 2009; Zhong et al. 2007). While indirect impacts such as these are observable and would contribute to the performance of parameter estimation, they are also likely to be situation specific and, in the case of moist convection, highly nonlinear. For moist convection in particular, the model response to changes in parameters may be quite erratic and thereby violate the simplicity requirement.

With only a single case and a limited number of ensemble members, we focus our evaluation on the direct 
impacts, as revealed through horizontal averages across the inner domain in areas free of simulated precipitation (Fig. 1). Such horizontally averaged impacts should be qualitatively consistent from case to case. This strategy excludes locations under the immediate influence of moist convection and averages across locally driven mesoscale circulations such as sea breezes and mountain-valley breezes. The horizontal extent of the inner domain includes a wide range of geographical conditions, from the Gulf of Mexico to the Sierra Madre Oriental. In addition to all portions of domain 3 without precipitation, two other horizontal averages are computed. The first is that portion of the precipitation-free domain over the Gulf of Mexico, and the second is that portion of the domain covering eastern Texas, which is mostly precipitation free.

Model output intercomparison and diagnosis are carried out on the inner domain (with a resolution of $12 \mathrm{~km}$ ). For each model parameter and each averaging area, temperature, moisture, and wind speed are diagnosed. Plots of model variables as a function of parameter values address the issue of simplicity, with a linear relationship between variables and parameter values being ideal. Standard deviation computed from the single-parameter output, is a measure of the magnitude of the variability in the model output associated with a particular parameter. A small standard deviation for a particular parameter means a change of that parameter across its plausible range of uncertainty is manifested by only small changes in the measurable model output variables. Such a parameter would not be observable. Correlation computed from the multiparameter output indicates to what extent variations in a particular parameter control the model output variable and suggests whether the impact of the parameter is distinguishable from the impacts of other parameters. The EnKF adjusts parameters using covariance information, that is, correlation multiplied by the variances of parameter and model outputs. A small correlation between the measurable output variable and a particular parameter results in a small Kalman gain and little impact on parameter values through assimilation of observations. Correlation was also used as a diagnostic by Hacker and Snyder (2005) to examine the efficacy of assimilating some specific observations using EnKF.

\section{Sensitivity analysis}

Figures 3-4 show output related to temperature: standard deviation (Fig. 3) and correlation (Fig. 4). Both figures depict the lowest $3000 \mathrm{~m}$ to more clearly show shallow boundary layer impacts. All quantities are computed and displayed in model space; the area-mean heights of the model levels are provided along the $y$ axis. Above $3000 \mathrm{~m}$ (not shown), the variability of temperature is largest near the model top where both stratification and vertical grid spacing are very large. The variability emerges first for $V$ and $K_{v}$, both of which affect vertical mixing in highly stable situations such as are normally found in the stratosphere.

In the lower troposphere, the parameters produce particular sensitivity patterns associated with their role in the ACM2 vertical mixing scheme. The first five parameters (i.e., $p, P, 0.1 a, \mathrm{Ri}_{\text {crit }}$, and $b$ ) show differing amplitudes but broadly similar patterns in their sensitivities in Fig. 3. The overall patterns (first row) of these five parameters are driven primarily by sensitivities over land, as indicated by the similar patterns (and stronger signal) over eastern Texas (third row) and dissimilar patterns over water (second row). Sensitivities over land during the first day are weaker than those during the second day but share a similar diurnal pattern, while sensitivities over water evolve steadily during this episode. Among the five, $P$ and $0.1 a$ show weaker sensitivities. The five parameters all show repeated clawlike regions of large sensitivity over land centered around $2000 \mathrm{~m}$ during afternoon and evening but that first appear at $1000 \mathrm{~m}$. This maximum sensitivity area corresponds to the entrainment zone at the top of daytime PBL and the evening residual layer.

The middle panel shows sensitivity over the northwestern Gulf of Mexico. Because the PBL over the Gulf of Mexico tends to be weakly unstable, the pattern of sensitivity is similar to that over land during daytime, but without the diurnal cycle. The maximum positive sensitivity increases from 500 to over $1000 \mathrm{~m}$ during the course of the simulation, implying that the marine PBL is similarly growing. Ordinarily the marine PBL is fairly stable in height around 500-600 $\mathrm{m}$ in the northwest Gulf area, so this rise in PBL depth may indicate a shortcoming of the model. However, the winds were offshore during most of the 2-day period, so it is possible that the increase of PBL depth is real and is a response to offshore advection of a deeper continental PBL.

The similar pattern seen with $p, P, 0.1 a, \mathrm{Ri}_{\text {crit }}$, and $b$ means changes of them alter the vertical mixing in similar regions during daytime. The parameter $p$ determines the value of the local eddy vertical mixing coefficient within the convective PBL, with larger $p$ leading to smaller vertical mixing. Weak vertical mixing, including reduced heat transport from the surface to the atmosphere and reduced entrainment at the top of the PBL, should produce a cooler PBL. Meanwhile, the reduced PBL height and reduced mixing from below should have a warming effect in the thin layer of air at the top of the PBL and the bottom of the free troposphere, sometimes called the entrainment layer. Being thin, the temperature sensitivity here can be much larger than 

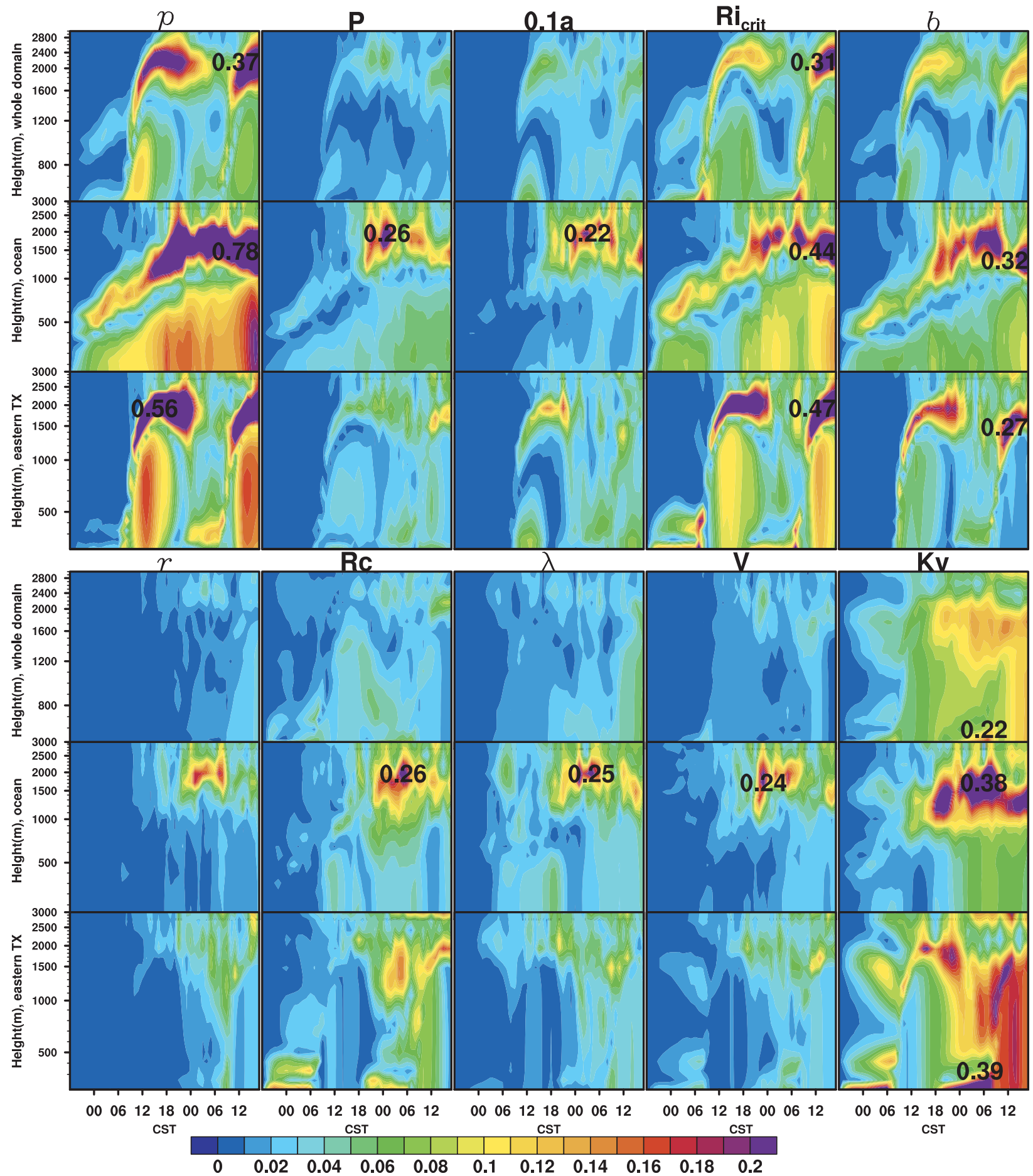

FIG. 3. Time-height sections of standard deviation of horizontally averaged potential temperature with respect to vertical mixing parameters (see column labels) over the whole inner domain, the water portion, and eastern TX (see row labels) in single-parameter model runs. Grid points with precipitation are not included in the calculations. Calculations are performed in model eta coordinates and labeled according to average altitude of the eta surfaces. The bottom corresponds to the eta surface adjoining the ground or water. Maximum values are labeled when they exceed $0.2 \mathrm{~K}$. 

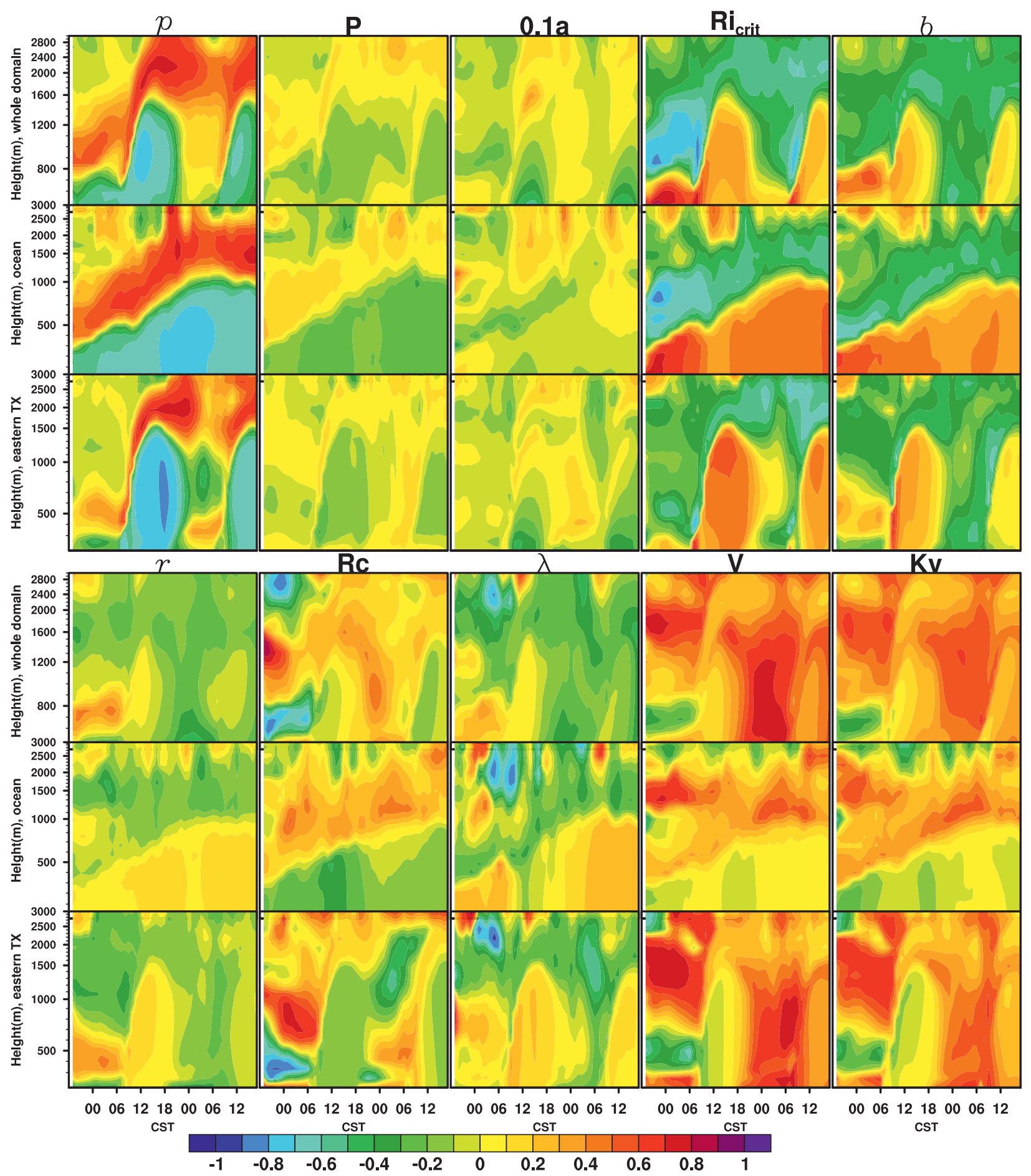

FIG. 4. Time-height sections of correlation of horizontally averaged potential temperature with respect to vertical mixing parameters (see column labels) over the whole inner domain, the water portion, and eastern TX (see row labels) from multiparameter runs. Organized as in Fig. 3.

within the well-mixed portion of the daytime PBL where thermodynamic changes are spread over a larger depth. The negative correlation between $p$ and temperature within the daytime PBL and the positive correlation at the top of the PBL (Fig. 4) are consistent with smaller mixing caused by larger $p$. Figure 5a shows the overall effect on the vertical temperature profile when $p$ alone is allowed to vary. The variability of temperature in the 

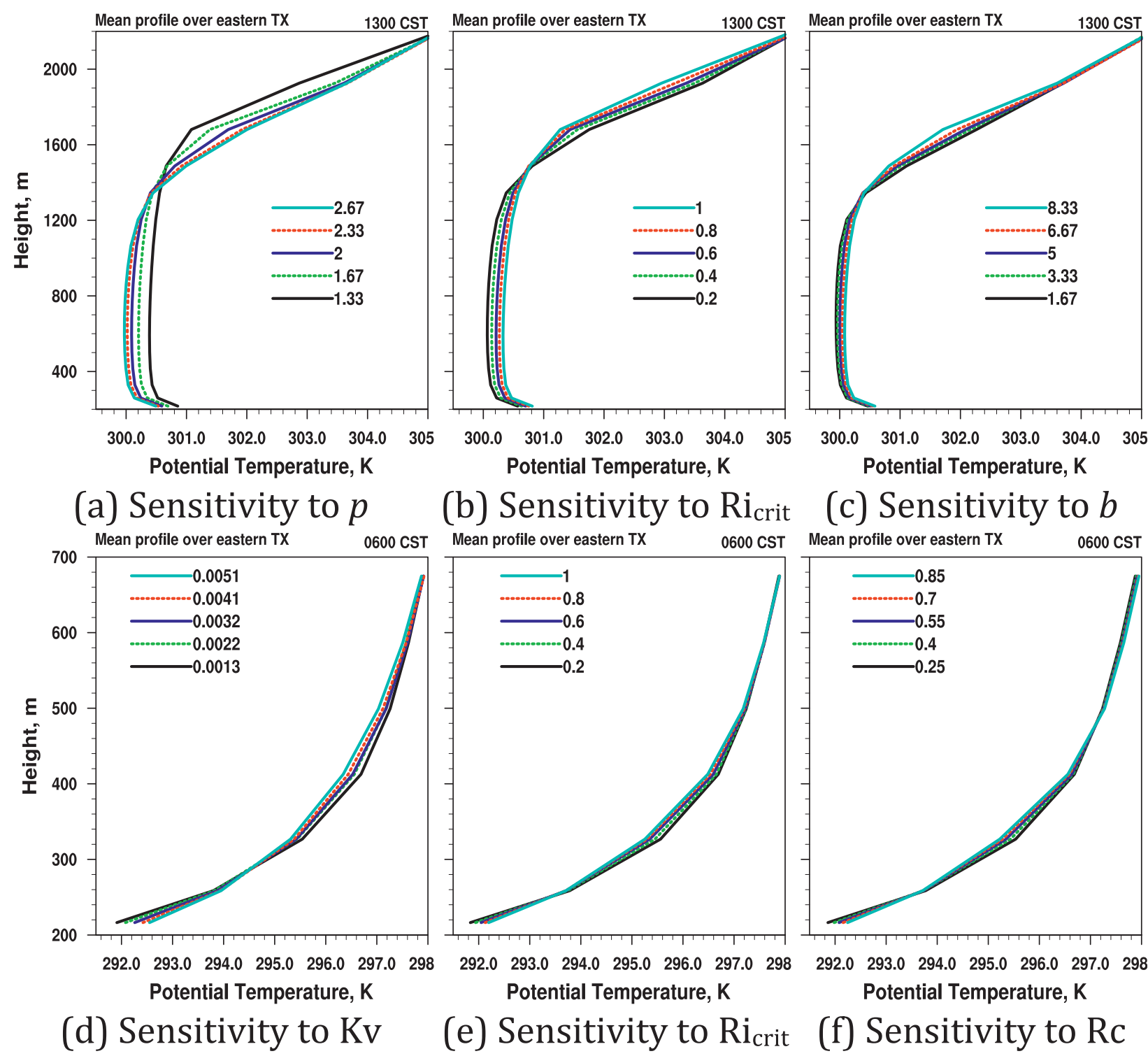

(b) Sensitivity to $\mathrm{Ri}_{\text {crit }}$

(c) Sensitivity to $b$
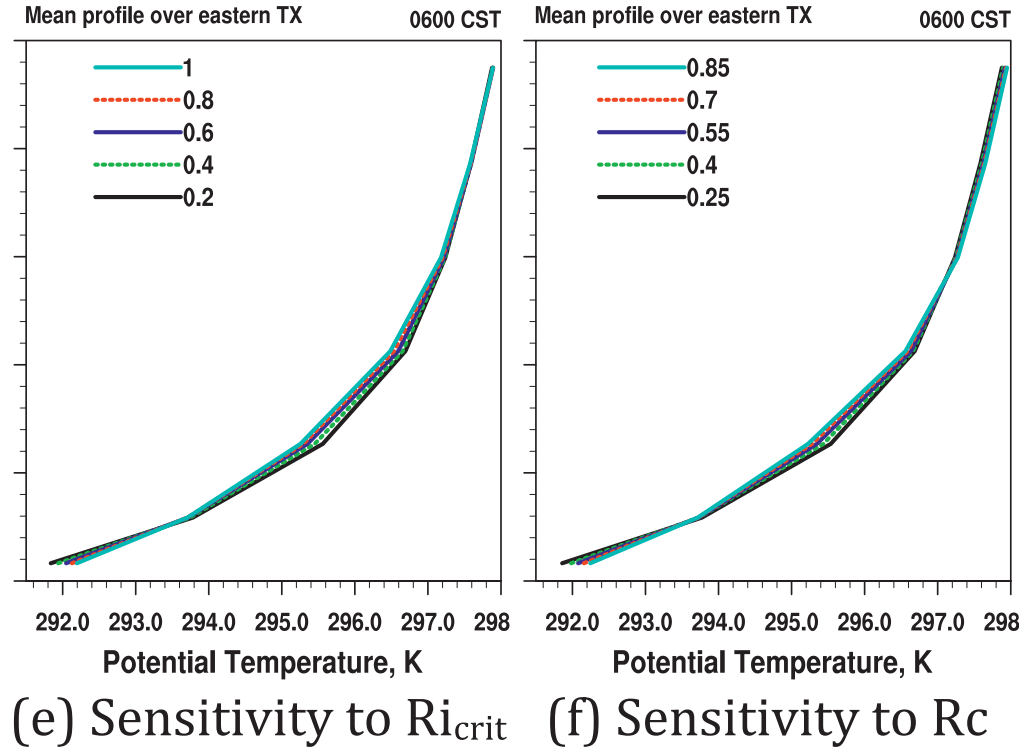

FIG. 5. Mean profile over eastern Texas at (top) 1300 CST 30 Aug 2006 and (bottom) 0600 CST 30 Aug 2006 due to different parameter values from single-parameter runs for the parameters giving the largest sensitivities.

daytime PBL associated with $p$ (Fig. 3) is the largest among all the parameters. The standard deviation of temperature in the PBL is as high as $0.6^{\circ} \mathrm{C}$ at the top of the PBL over eastern Texas. This means that the parameter $p$ plays the most important role in controlling the vertical mixing during the daytime.

The $\mathrm{Ri}_{\text {crit }}$ is the threshold value for detecting the top of PBL, and $b$ represents the excess buoyancy of surfacebased parcels. Both of them are used to determine the PBLH under convective conditions. Larger values of them lead to higher PBLH, causing stronger local and nonlocal mixing. Thus, their correlation with temperature is opposite that of $p$ in the PBL: negative at the top of the
PBL and positive within the daytime PBL. The $\mathrm{Ri}_{\text {crit }}$ tends to produce a larger sensitivity (Fig. 3) than $b$, and $\mathrm{Ri}_{\text {crit }}$ also affects low-level temperatures at night. Figures $5 \mathrm{~b}, \mathrm{c}$ confirm that larger values of $\mathrm{Ri}_{\text {crit }}$ and $b$ are associated with deeper PBLs.

The parameter $0.1 a$ is used to determine the portion of mixing due to nonlocal transport (i.e., $f_{\text {conv }}$ ). Larger $f_{\text {conv }}$ leads to lower temperatures in the lower part of the PBL and higher temperature in the upper part (Pleim 2007a). Altering $0.1 a$ would have the same effect since the relationship is monotonic between $0.1 a$ and $f_{\text {conv }}$. Such an effect is seen in the positive correlation of $0.1 a$ with temperature in the upper PBL and negative correlation in the 
lower PBL (Fig. 4). The vertical correlation dipole is shallower than with those parameters discussed previously, which involve major sensitivities at and above the top of the PBL.

The parameter $P$ also has a somewhat different vertical profile of sensitivity. It determines the relative magnitudes of mixing of heat and constituents versus momentum, with larger $P$ leading to smaller mixing of heat relative to momentum. The correlation between $P$ and temperature is negative within most of the daytime PBL, but positive at the ground and in the entrainment zone.

Of the other five parameters, only Rc and $K_{v}$ have significant impacts on temperature. Both have their largest effects at night, with positive correlations with surface temperatures and negative correlations with temperatures at 300-400 m during the nighttime. This is consistent with larger values of both parameters leading to stronger vertical mixing. An effect similar in sign but smaller in magnitude is found with $\mathrm{Ri}_{\text {crit }}$ for nighttime temperature. The largest sensitivity (standard deviation of $0.4^{\circ} \mathrm{C}$ ) of nighttime temperature is associated with $K_{v}$.

The lower row of Fig. 5 shows the mean profile over eastern Texas at 0600 CST 30 August due to different parameter values for the three parameters that give the largest sensitivity during nighttime (i.e., $K_{v}, \mathrm{Ri}_{\text {crit }}$, and $\mathrm{Rc}$ ) from single-parameter runs. These profiles demonstrate their similar functions during nighttime. The surface temperatures almost linearly depend on these parameters. The effects of $\mathrm{Ri}_{\text {crit }}$, and $\mathrm{Rc}$ are limited to the vicinity of the PBL while $K_{v}$ also affects the mixing in the upper troposphere.

Figures 6-7 show the sensitivities and correlations related to water vapor mixing ratio. As with potential temperature, the largest sensitivities are found within the boundary layer, particularly in the entrainment zone at the top of the boundary layer. Sensitivities to moisture tend to be largest over the water portion of the domain. The correlations with mixing ratio also retain their sign from daytime to nighttime, probably because latent heat fluxes are upward from the surface throughout the diurnal cycle while the sensible heat flux changes sign over land from daytime to nighttime. Following the first growth of the convective boundary layer, the correlations with mixing ratio change very little with time. In general, the same parameters are important for both potential temperature and mixing ratio, except that $K_{v}$ 's impact on mixing ratio is much smaller than that of some of the other parameters.

The sign of the mixing ratio correlations during daytime is almost uniformly opposite in sign to the potential temperature correlations. This is consistent with variations of the PBL parameters controlling the vertical growth of the PBL and entrainment from the free troposphere. Air parcels entrained from the free troposphere tend to bring with them relatively high values of potential temperature and relatively low values of mixing ratio.

The mixing variations in the upper troposphere due to changes in $K_{v}$ lead to different vertical distribution of both temperature and water vapor, then to different cloud patterns, and thus different shortwave radiation amounts. Thus, the mixing variation due to $K_{v}$ in the upper troposphere causes a complicated nonlinear feedback throughout the atmosphere. Unlike other parameters (e.g., $p, \mathrm{Ri}_{\text {crit }}$, and $b$ ) whose sensitivity on the second day is similar to that on the first, $K_{v}$ has different sensitivity during daytime of the second day due to the cloud effects. The correlation between $K_{v}$ and temperature in the lower troposphere shown in Fig. 4 on the second day cannot be explained by the direct local impacts of $K_{v}$. Since $\lambda$ and $V$ also affect mixing in the free troposphere, their correlations with PBL meteorology parameters are also complicated by cloud effects.

Figures 8-9 show the sensitivities and correlations related to wind speed. Wind sensitivities tend to have the same signs and relative magnitudes as the potential temperature sensitivities, since both potential temperature and wind speed tend to increase upward and are affected in similar ways by vertical mixing. The same parameters are associated with large sensitivities with both wind and temperature (i.e., $p$ and $\mathrm{Ri}_{\text {crit }}$ for daytime, Rc and $K_{v}$ for nighttime). One notable difference between the temperature and wind sensitivities is that the wind sensitivities tend to have more "noise," with rapid variations of sensitivity that are not consistent from day to day. So temperature sensitivities are more systematic than wind sensitivities. Another difference worth mentioning is that Rc shows the largest sensitivity for nighttime wind speed (standard deviation of $0.52 \mathrm{~m} \mathrm{~s}^{-1}$ ) and highly correlates with nighttime wind speed (up to 0.95). It is more important to nighttime wind speed than $K_{v}$ and dominates over other parameters.

\section{Identifiability assessment}

The three dimensions of identifiability are observability, simplicity, and distinguishability. All three of these dimensions will in general be sensitive to the specific observations available for assimilation, but two parameters can be discarded immediately without consideration of the observation network. The parameter $r$ has low sensitivities at all levels and times over its expected range, and thus will be much less observable than the other parameters. The parameter $b$ has moderate sensitivities, but the correlation patterns closely match those of $p$. Thus, $b$ and $p$ are not distinguishable, and $b$, having weaker sensitivities, should be discarded. 

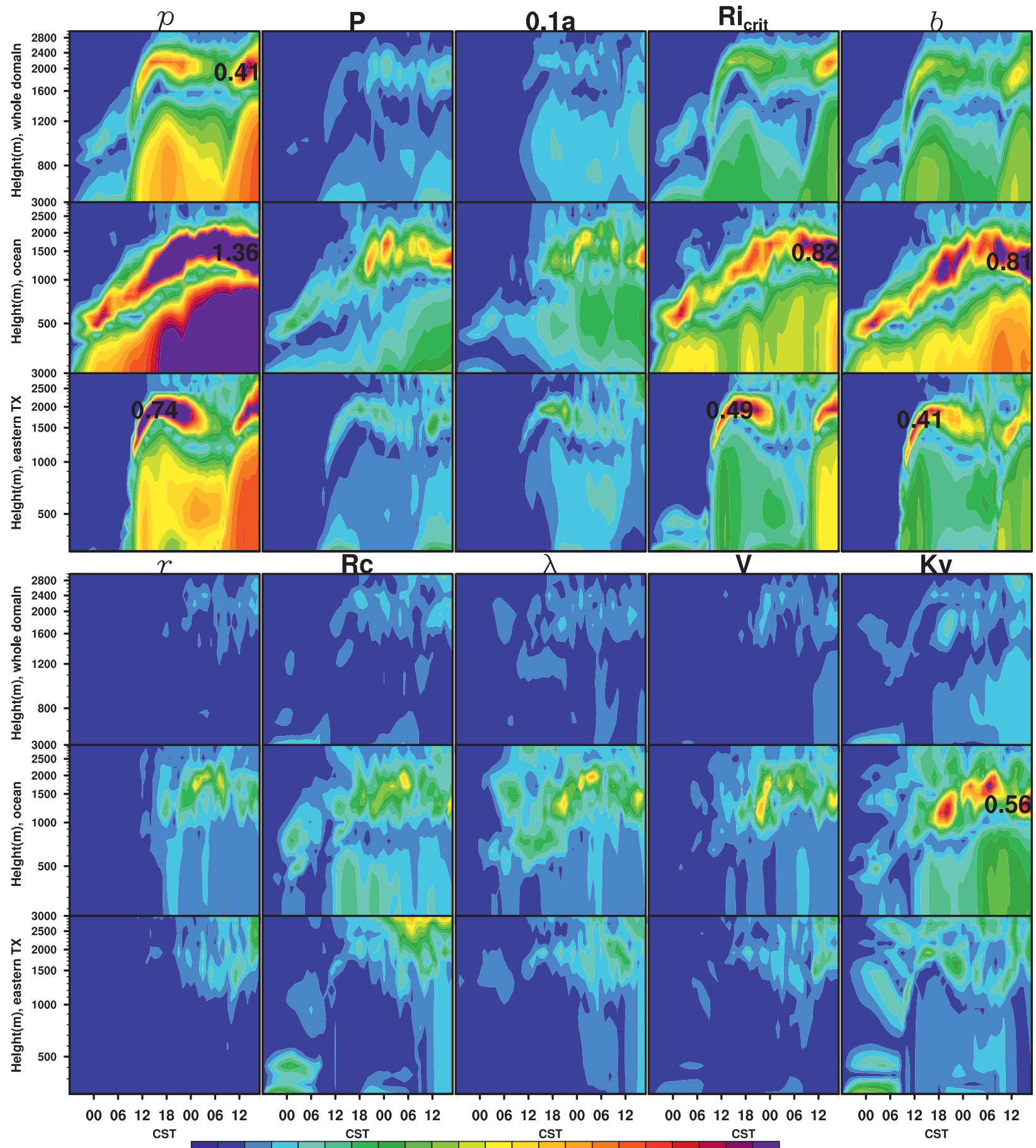

$\begin{array}{lllllllllll}0 & 0.04 & 0.08 & 0.12 & 0.16 & 0.2 & 0.24 & 0.28 & 0.32 & 0.36 & 0.4\end{array}$

FIG. 6. Time-height sections of standard deviation of horizontally averaged water vapor mixing ratio with respect to vertical mixing parameters (see column labels) over the whole inner domain, the water portion, and eastern TX (see row labels) in single-parameter model runs. Grid points with precipitation are not included in the calculations. Maximum values are labeled when they exceed $0.4 \mathrm{~g} \mathrm{~kg}^{-1}$.

Among the remaining eight parameters, some are more important during daytime while others are more important during nighttime. Because most parameter correlations have substantial vertical structure, which varies from parameter to parameter, observations of profiles of temperature, moisture, and wind in the PBL would allow for much greater distinguishability than surface observations alone. The most common source for observed 

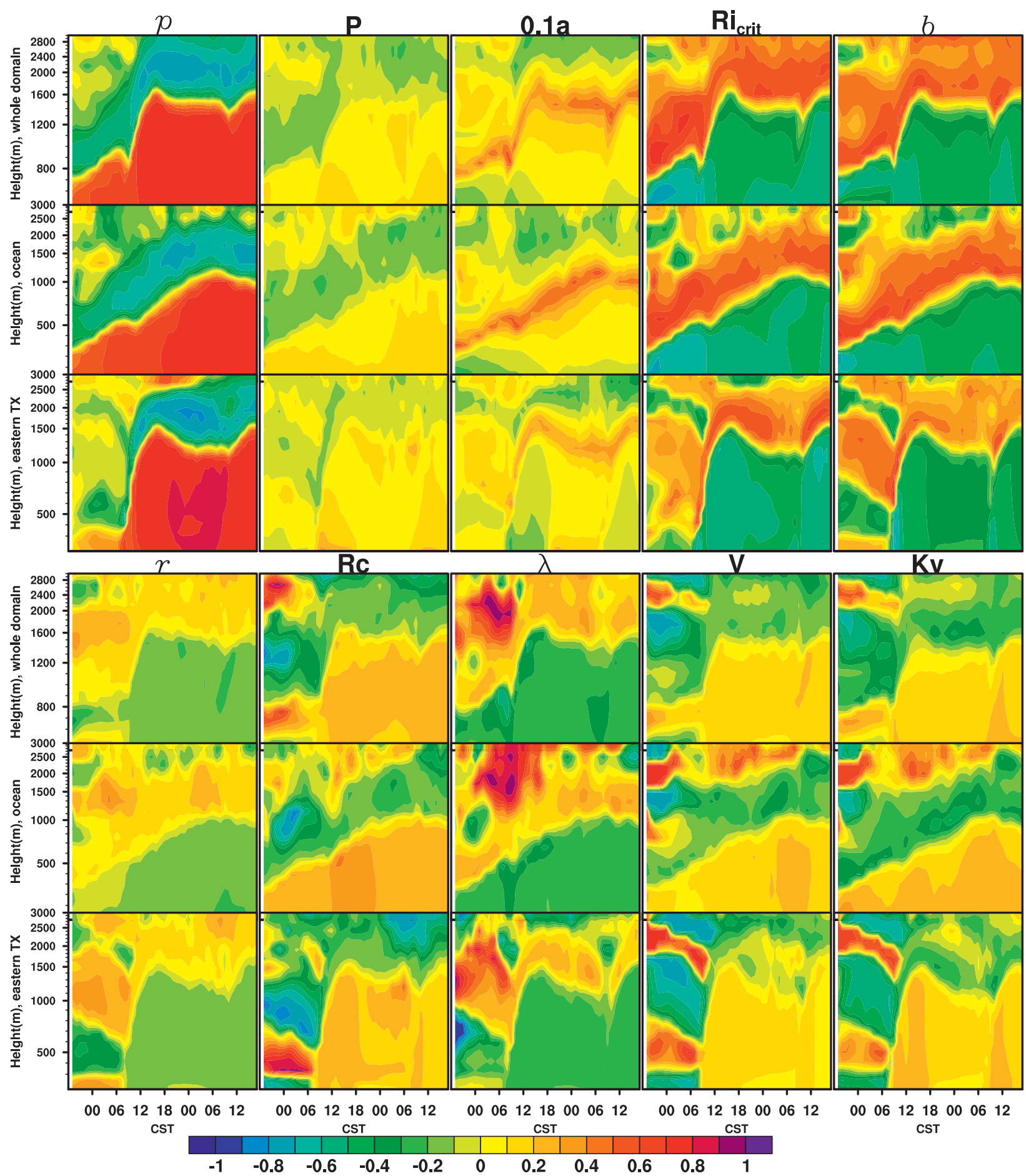

FIG. 7. Time-height sections of correlation of horizontally averaged water vapor mixing ratio with respect to vertical mixing parameters (see column labels) over the whole inner domain, the water portion, and eastern TX (see row labels) from multiparameter runs. Organized as in Fig. 6.

temperature, moisture, and wind profiles are rawinsondes, but in the central and eastern United States the rawinsonde launch times are not at the times of maximum sensitivity. The efficacy of assimilating rawinsonde data to adjust parameters may be largely confined to effects caused by mixing ratio observations, since mixing ratio sensitivities are relatively uniform throughout the diurnal cycle. 

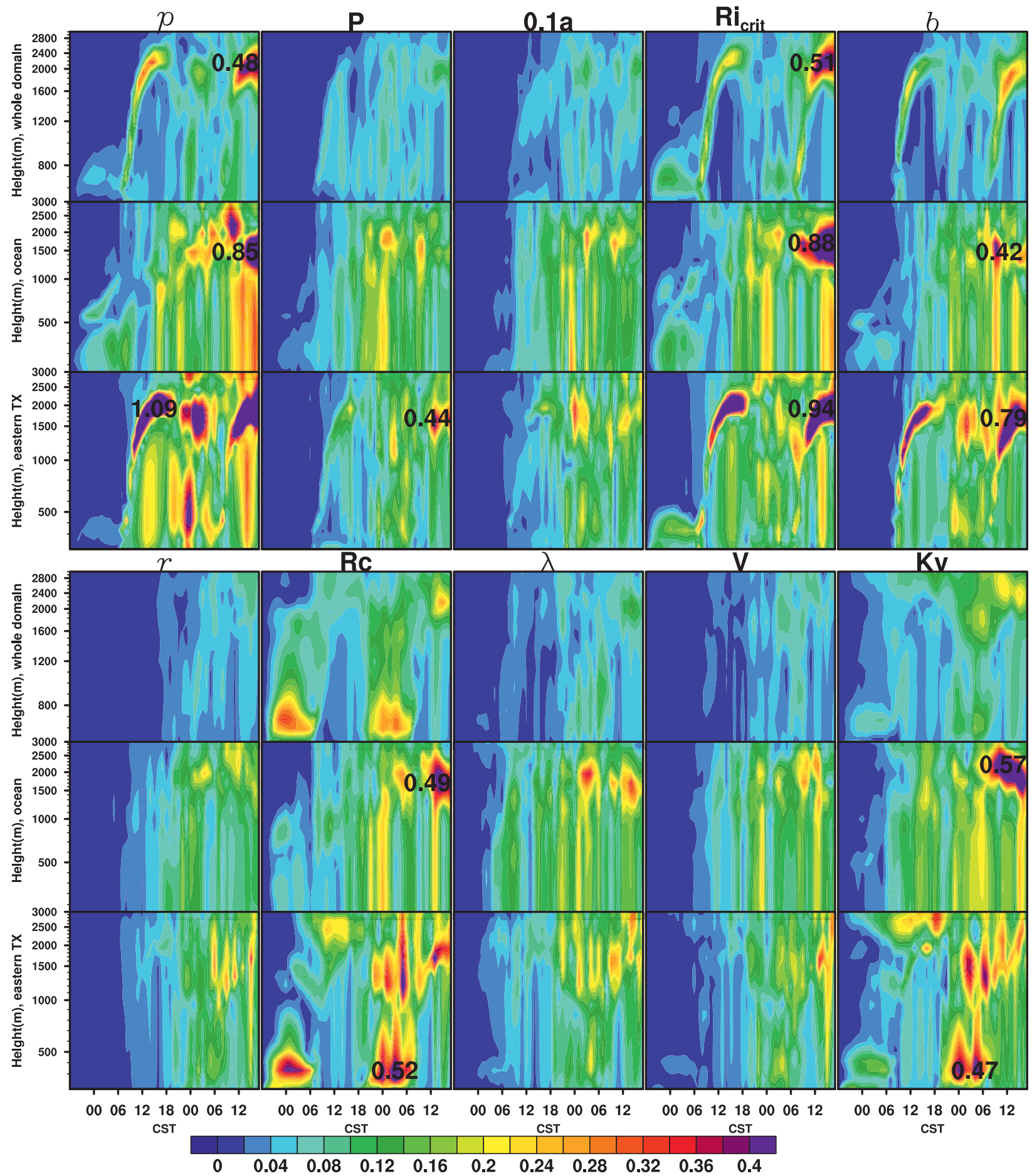

FIG. 8. Time-height sections of standard deviation of horizontally averaged wind speed with respect to vertical mixing parameters (see column labels) over the whole inner domain, the water portion, and eastern TX (see row labels) in single-parameter model runs. Grid points with precipitation are not included in the calculations. Maximum values are labeled when they exceed $0.4 \mathrm{~m} \mathrm{~s}^{-1}$.

Unlike rawinsonde observations, radar wind profiler observations are effectively continuous and, when coupled with the Radio-Acoustic Sounding Systems (RASS), provide virtual temperature profiles as well.
At night, the greatest wind sensitivity and highest correlation within boundary layer profiler range is with $\mathrm{Rc}$ (Fig. 8). The standard deviation of wind speed is approximately $0.52 \mathrm{~m} \mathrm{~s}^{-1}$ at the level of the nighttime 

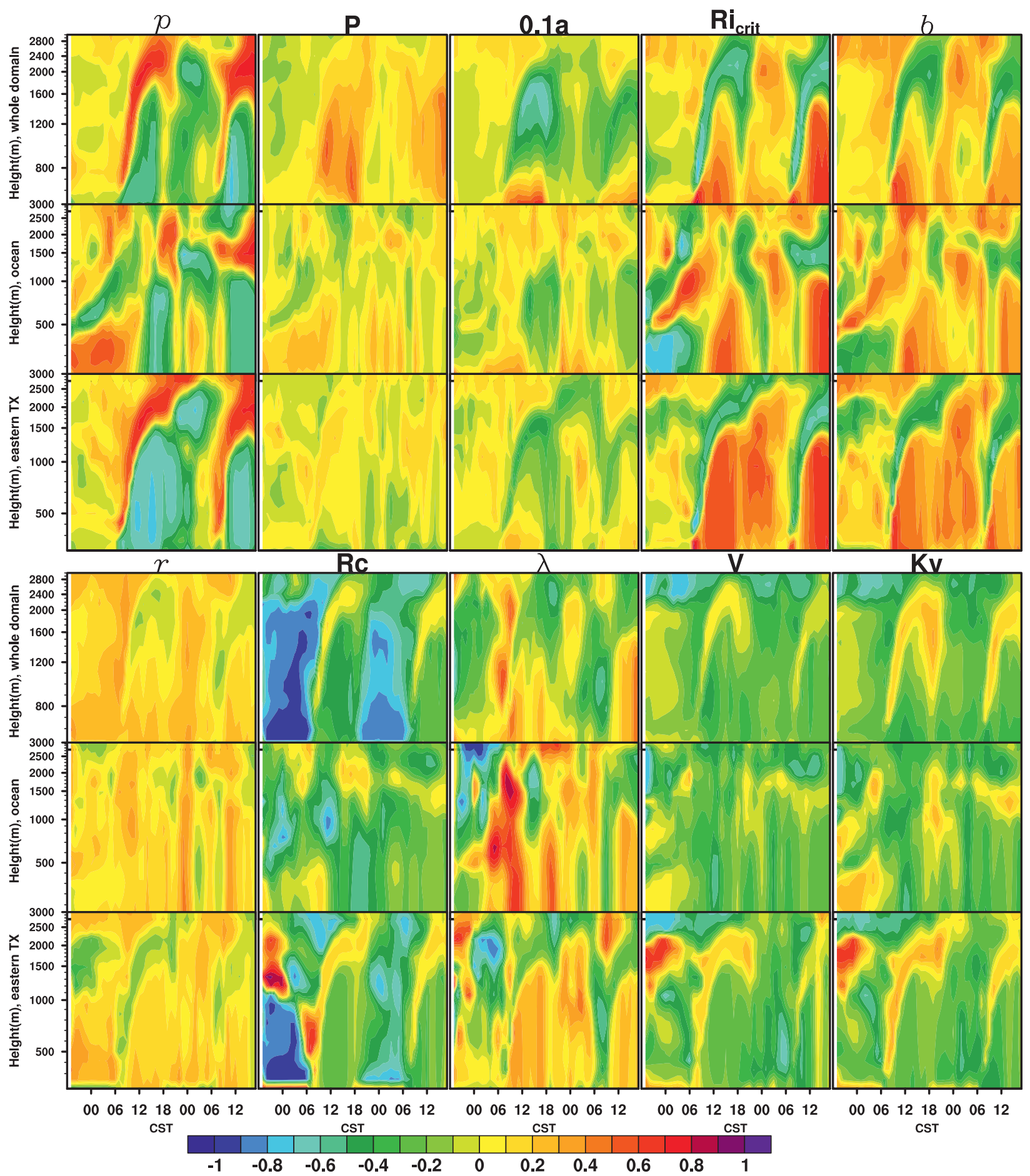

FIG. 9. Time-height sections of correlation of horizontally averaged wind speed with respect to vertical mixing parameters (see column labels) over the whole inner domain, the water portion, and eastern TX (see row labels) from multiparameter runs. Organized as in Fig. 8.

low-level jet over eastern Texas. Sensitivity to Rc during the daytime is very weak. The parameter $K_{v}$ is associated with somewhat lower sensitivities and much weaker correlations and might not be distinguishable from Rc at night, but $K_{v}$ also has substantial sensitivities during the day.

For daytime sensitivity, the most identifiable parameter is $p$. Wind speed has a large negative correlation 
with $p$ within the daytime PBL and a very large positive correlation at the top of the daytime PBL. Wind speed also has substantial sensitivity to $\mathrm{Ri}_{\text {crit }}$, and its sensitivity in late afternoon and evening is distinguishable from $p$. Other parameters, albeit with weaker sensitivities, are distinguishable because of their vertical profiles. Large values of $0.1 a$ increase the daytime wind speed in the lowest $200 \mathrm{~m}$ and in the entrainment zone and decrease it within the upper half of the PBL. The sensitivity to $P$ is weak, but the correlations have a unique structure, with the same sign in the PBL as in the entrainment zone.

Thus, in order of likely applicability for parameter estimation through assimilating wind profiler data, the most identifiable parameters are $\mathrm{Rc}$, and $p$, followed by $K_{v}, 0.1 a, \mathrm{Ri}_{\text {crit }}$, and $P$. The exact number of parameters to be retained depends on the characteristics of the observation network.

If only surface observations are to be assimilated into the numerical model, the mixing parameters to be estimated should be those that produce large sensitivities at the surface. For wind speed, the largest parameter impacts are associated with $K_{v}$ (Fig. 8), with negative correlations at night and positive correlations during the day. Distinguishable from $K_{v}$ are $p$, with substantial correlations (positive) during daytime only; $\mathrm{Ri}_{\text {crit }}$, with peaks in sensitivity just before dawn and late in the afternoon; and Rc, with sensitivity confined to the nighttime. For surface temperature, $K_{v}$ and $\mathrm{Ri}_{\text {crit }}$ both produce large sensitivities at night, with somewhat overlapping temperature patterns. In contrast, $p$ produces substantial sensitivities during the daytime only. So if surface observations are to be assimilated, the best parameters to be estimated should be $K_{v}$ and $p$, followed by $\mathrm{Ri}_{\text {crit }}$.

So far, only the distinguishability and observability dimensions of identifiability have been explicitly considered. To address simplicity, Fig. 10 shows domain-averaged surface temperature anomalies for those parameters with the strongest surface temperature identifiability. The right column shows results from single-parameter runs; for the most part, the mean temperatures vary smoothly as the parameter values change, implying a single optimal parameter value for a given surface temperature. Over land, $p$ shows an irregular variation of mean temperature at lower $p$ values, but the output from the multiparameter runs presents a larger number of realizations and suggests that the temperature dependence on $p$ would be expected to be monotonic and positive over land, negative over water. The $\mathrm{Ri}_{\text {crit }}$ is more troubling; over land the singleparameter runs suggest a local temperature minimum at $\mathrm{Ri}_{\text {crit }}=0.4$, and the multiparameter runs likewise suggest that temperature may be warmer for both large and small values of $\mathrm{Ri}_{\text {crit }}$. Different values of $\mathrm{Ri}_{\text {crit }}$ would provide equally good matches to surface temperature. Thus, if limited to surface observations, $\mathrm{Ri}_{\text {crit }}$ may not be identifiable because of a lack of simplicity. Further investigation is needed to determine whether $\mathrm{Ri}_{\text {crit }}$ would be identifiable through induced variations of temporal behavior of temperature or through wind variations.

\section{Conclusions}

Simulations of PBL meteorology may be biased because of the uncertainties in PBL parameterization schemes. Estimation of the optimal values for the parameters used in PBL schemes may allow significant improvements in the representation of vertical mixing within and above the PBL. For parameter estimation to be successful, the parameters must be identifiable, meaning that they must have a detectible impact on verifiable aspects of the model behavior, the impact must be a simple function of the parameter values, and the impact must be clearly distinguishable from impacts caused by other parameter variations. In this study, 10 parameters in the ACM2 PBL scheme amenable to parameter estimation are first identified. Plausible physical bounds for each parameter are given based on previous theory or observations.

Multiple sets of model simulations were performed to test the sensitivity of the WRF model to the 10 ACM2 parameters in their plausible physical bounds. The parameter $p$ (the exponent in the formulation of boundary layer scaling vertical eddy diffusivity) is shown to play the most important role in controlling the vertical mixing during the daytime among the 10 parameters tested. Changes in $p$ within its plausible range cause variations of more than $1^{\circ} \mathrm{C}$ within and just above the daytime PBL. The parameter $\mathrm{Ri}_{\text {crit }}$ (the threshold value for detecting the top of PBL) is shown to cause the second largest variability of temperature in the daytime PBL. The minimum value of eddy diffusivity $K_{v}$ is shown to cause the largest variations of temperature $\left(\sim 0.8^{\circ} \mathrm{C}\right)$ in nighttime PBL, followed by Rc (a critical Richardson number that defines the onset of turbulence). Because of the similarity of processes affecting the profiles of potential temperature, moisture, and wind speed, the parameters that cause the largest variability of temperature also cause largest variability of moisture and wind speed, except that Rc causes the largest variability of wind speed $\left(>1 \mathrm{~m} \mathrm{~s}^{-1}\right)$ during nighttime around the level of the nighttime lowlevel jet.

All of the examined ACM2 parameters affect the vertical profiles of temperature, moisture, and wind speed. Thus, profiler-type observations contain the best information about those parameters. Assimilating radar wind profiler data with RASS with enough frequency would have the best chance of successfully calibrating those parameters and improving the simultaneous state 
Multi-parameter runs (10 parameters perturbed simultaneously)
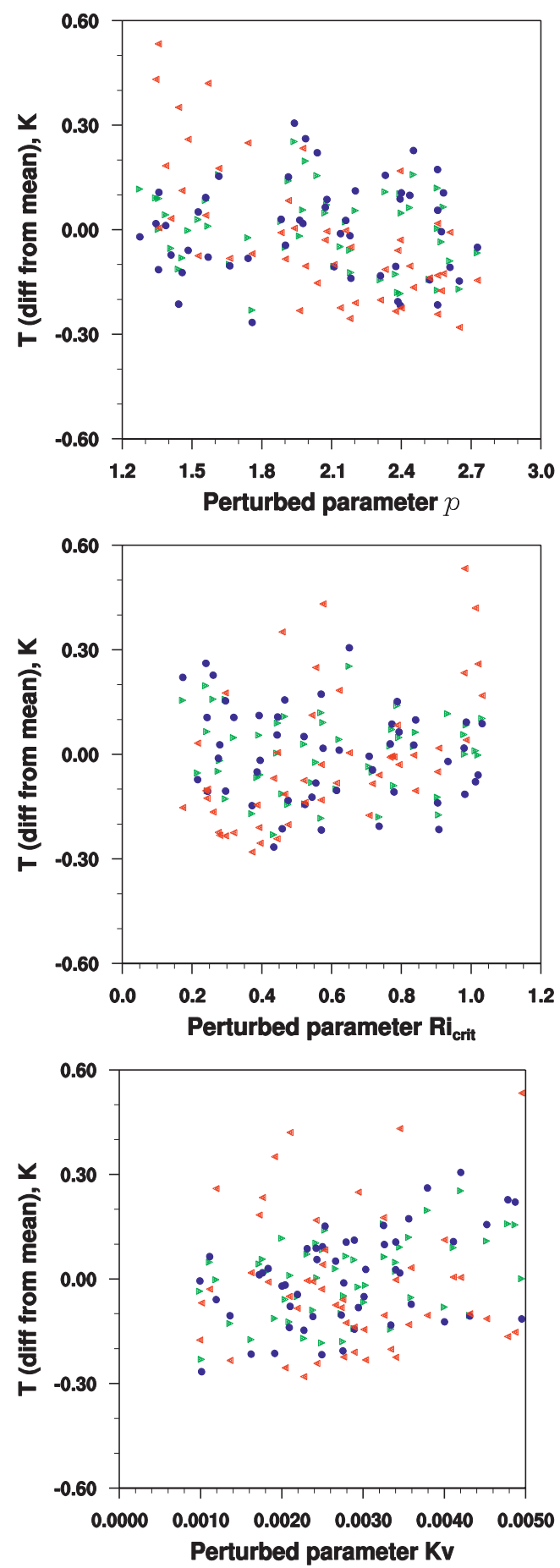

Single-parameter runs (perturb 1 parameter at 1 time)
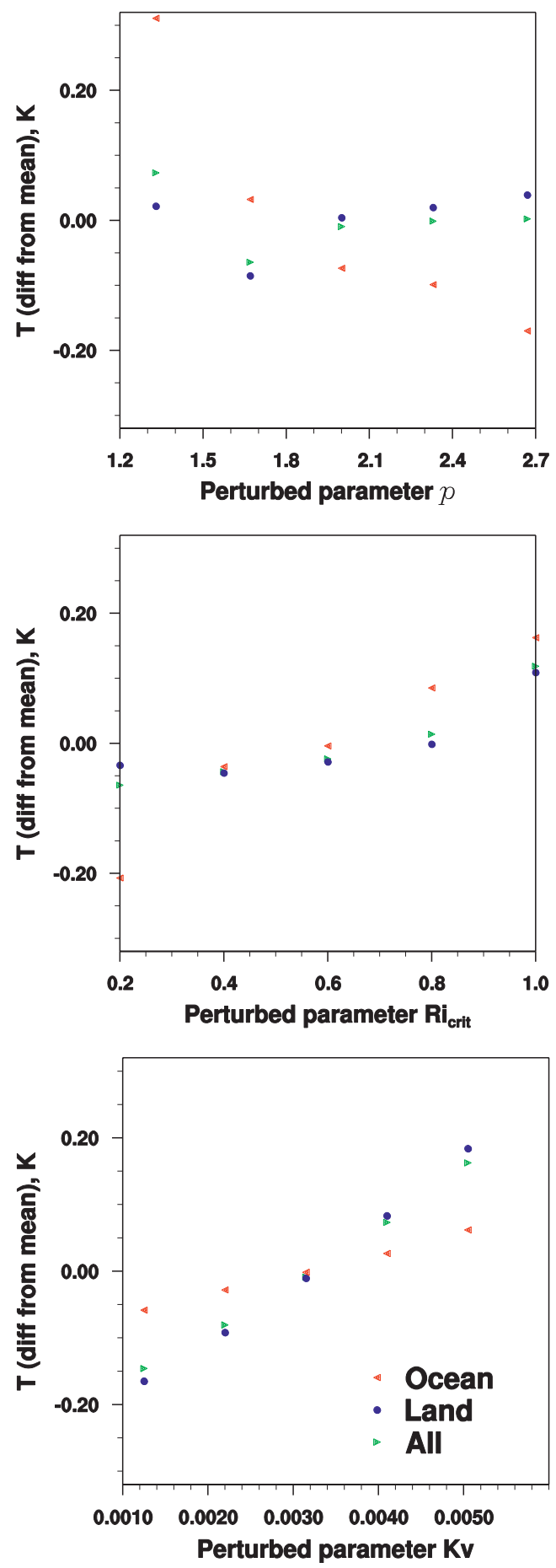

FIG. 10. Scatterplots showing domain-averaged (excluding regions with precipitation) values of temperature at 1700 CST 31 Aug 2006 as a function of parameter values (green). (left) Results are from multiparameter simulations; (right) results are from single-parameter simulations. Averages restricted to precipitation-free ocean (red) and land (blue) are also shown. Parameters are (top) $p$, (middle) $\mathrm{Ri}_{\text {crit }}$, and (bottom) $K_{v}$. 
estimation. If such data are assimilated, the two most identifiable parameters are Rc and $p$. If no profile data are available and only surface observations are to be assimilated, the two most identifiable parameters are $K_{v}$ and $p$. These results pertain only to direct impacts of the parameters; to the extent that changes in PBL structure affect moist convection and other observable aspects of the atmosphere, the amenability of certain parameters to parameter estimation may be quite different from the circumstances presented here.

The sensitivity results reported here were determined from model runs covering a particular geographical area during a particular time interval. As can be seen from comparison of the sensitivities over land and over water, the absolute sensitivities will depend upon the meteorological and geographical circumstances. However, because the greatest sensitivities are associated with the same parameters whether over land or over water, the relative importance of particular parameters appears to be robust to the meteorological and geographical setting. The absolute and relative sensitivities also depend directly upon the chosen plausible ranges for each parameter; changes in such ranges would produce corresponding absolute and relative changes in the sensitivities.

The initial results of parameter estimation data assimilation experiments using ACM2 in WRF, with Rc and $p$ as the adjustable parameters, are reported in $\mathrm{Hu}$ et al. (2010b).

Acknowledgments. This work was supported by the State of Texas through a contract from the Houston Advanced Research Center, the Texas Environmental Research Consortium, and the Texas Commission on Environmental Quality.

\section{REFERENCES}

Aksoy, A., F. Zhang, and J. W. Nielsen-Gammon, 2006a: Ensemblebased simultaneous state and parameter estimation with MM5. Geophys. Res. Lett., 33, L12801, doi:10.1029/2006GL026186.

$\ldots, \ldots$, and _ 2006b: Ensemble-based simultaneous state and parameter estimation in a two-dimensional sea-breeze model. Mon. Wea. Rev., 134, 2951-2970.

Chen, F., and J. Dudhia, 2001: Coupling an advanced land surfacehydrology model with the Penn State-NCAR MM5 modeling system. Part I: Model implementation and sensitivity. Mon. Wea. Rev., 129, 569-585.

Dudhia, J., 1989: Numerical study of convection observed during the winter monsoon experiment using a mesoscale twodimensional model. J. Atmos. Sci., 46, 3077-3107.

Foken, T., 2006: 50 years of the Monin-Obukhov similarity theory. Bound.-Layer Meteor., 119, 431-447, doi:10.1007/s10546-0069048-6.

Hacker, J. P., and C. Snyder, 2005: Ensemble Kalman filter assimilation of fixed screen-height observations in a parameterized PBL. Mon. Wea. Rev., 133, 3260-3275.
Holtslag, A. A. M., and B. A. Boville, 1993: Local versus nonlocal boundary-layer diffusion in a global climate model. J. Climate, 6, 1825-1842.

Hong, S.-Y., J. Dudhia, and S.-H. Chen, 2004: A revised approach to ice-microphysical processes for the bulk parameterization of cloud and precipitation. Mon. Wea. Rev., 132, 103-120.

Hu, X.-M., J. W. Nielsen-Gammon, and F. Zhang, 2010a: Evaluation of three planetary boundary layer schemes in the WRF model. J. Appl. Meteor. Climatol., in press.

_ , F. Zhang, and J. W. Nielsen-Gammon, 2010b: Ensemblebased simultaneous state and parameter estimation for treatment of mesoscale model error: A real-data study. Geophys. Res. Lett., 37, L08802, doi:10.1029/2010GL043017.

Jankow, I., W. A. Gallus Jr., M. Segal, B. Shaw, and S. E. Koch, 2005: The impact of different WRF model physical parameterizations and their interactions on warm season MCS rainfall. Wea. Forecasting, 20, 1048-1060.

Kondrashov, D., C.-J. Sun, and M. Ghil, 2008: Data assimilation for a coupled ocean-atmosphere model. Part II: Parameter estimation. Mon. Wea. Rev., 136, 5062-5076.

Miao, J.-F., K. Wyser, D. Chen, and H. Ritchie, 2009: Impacts of boundary layer turbulence and land surface process parameterizations on simulated sea breeze characteristics. Ann. Geophys., 27, 2303-2320.

Mlawer, E. J., S. J. Taubman, P. D. Brown, M. J. Iacono, and S. A. Clough, 1997: Radiative transfer for inhomogeneous atmospheres: RRTM, a validated correlated- $k$ model for the longwave. J. Geophys. Res., 102, 16 663-16 682.

Navon, I. M., 1997: Practical and theoretical aspects of adjoint parameter estimation and identifiability in meteorology and oceanography. Dyn. Atmos. Oceans, 27, 55-79.

Nielsen-Gammon, J. W., F. Zhang, A. M. Odins, and B. Myoung, 2005: Extreme rainfall in Texas: Patterns and predictability. Phys. Geogr., 26, 340-364.

Parrish, D. D., and Coauthors, 2009: Overview of the Second Texas Air Quality Study (TexAQS II) and the Gulf of Mexico Atmospheric Composition and Climate Study (GoMACCS). J. Geophys. Res., 114, D00F13, doi:10.1029/2009JD011842.

Pleim, J. E., 2007a: A combined local and nonlocal closure model for the atmospheric boundary layer. Part I: Model description and testing. J. Appl. Meteor. Climatol., 46, 1383-1395.

_ 2007b: A combined local and nonlocal closure model for the atmospheric boundary layer. Part II: Application and evaluation in a mesoscale meteorological model. J. Appl. Meteor. Climatol., 46, 1396-1409.

, and J. S. Chang, 1992: A non-local closure model for vertical mixing in the convective boundary layer. Atmos. Environ., 26A, 965-981.

Tong, M., and M. Xue, 2008: Simultaneous estimation of microphysical parameters and atmospheric state with simulated radar data and ensemble square root Kalman filter. Part I: Sensitivity analysis and parameter identifiability. Mon. Wea. Rev., 136, 1630-1648.

Troen, I., and L. Mahrt, 1986: A simple model of the atmospheric boundary layer: Sensitivity to surface evaporation. Bound.Layer Meteor., 37, 129-148.

Zhong, S., H. In, and C. Clements, 2007: Impact of turbulence, land surface, and radiation parameterizations on simulated boundary layer properties in a coastal environment. J. Geophys. Res., 112, D13110, doi:10.1029/2006JD008274.

Zupanski, D., and M. Zupanski, 2006: Model error estimation employing an ensemble data assimilation approach. Mon. Wea. Rev., 134, 1337-1354. 